\title{
ALL SYMBOLS WORD GENERATED BY REGULAR EXPRESSION
}

\author{
Thinh D. Nguyen \\ Email: kosmofarmer@yandex.com
}

\begin{abstract}
We show that deciding whether a regular expression generates all symbols word.
\end{abstract}

\section{Problem STATEMENT}

Given an alphabet $\mathbf{A}$ and a regular expression, we want to check if this regular expression generate word containing all symbols in $\mathbf{A}$.

\section{PROOF OF HARDNESS}

3-SAT can be reduced to this problem. Given a CNFwith $m$ clauses $(\mathbf{A}, \mathbf{B}, \mathbf{C}, \mathbf{D}, \ldots)$ and $n$ variables $\left(x_{1}, x_{2}, \ldots, x_{n}\right)$, where each clause contains three literals, we can construct a corresponding regular expression on an alphabet with $m$ symbols; the regular expression will accept a word with all $m$ symbols if and only if the CNF is satisfiable. Specifically, the regular expression consists of $n$ blocks of the form $\left(a_{i} \mid b_{i}\right)$, where $a_{i}$ (resp. $b_{i}$ ) is a string of the symbols corresponding to exactly those clauses that contain $x_{i}$ (resp. $\neg x_{i}$ ). (If $a_{i}$ is empty, replace this by $b_{i}$ ?, and vice-versa.) For example, if the CNF is

$$
\left(x_{1} \vee \neg x_{2} \vee \neg x_{3}\right) \wedge\left(x_{4} \vee x_{1} \vee x_{2}\right) \wedge\left(\neg x_{4} \vee x_{5} \vee \neg x_{2}\right)
$$

Then the regular expression is

$$
\text { (AB)?(B|AC)A?(B|C)C? }
$$

Any assignment of truth variables corresponds to a path through the regular expression, making a binary choice for each variable (resp., each block); a satisfying assignment (word) is one that makes every clause true (includes every symbol).

\section{CONCLUSION}

Garey and Johnson [1] shape their theory based on previous primal works of Cook, Levin and Karp. Johnson [2] moves on with the guide to this theory. As long as we study a mathematical conjecture, we should encourage ourselves to have moderate amount of labor hours on popular mathematics books like these. Then, reading some articles on theory of computing like [3] is a good practice. Having all those done, we could think of the ultimate final for all mathematics sciences.

\section{REFERENCE}

1. Michael R. Garey, David S. Johnson, Computers and Intractability: A Guide to the The ory of NP-Completeness

2. David S. Johnson, The NP-Comple teness Column: An Ongoing Guide

3. Phan Dinh Dieu, Le Cong Thanh, Le Tuan Hoa, Average Polynomial Time Complexity of Some NP-Complete Problems, Theor. Comput. Sci. 46(3): 219-237 (1986) 